\title{
Ultrastructural Morphology of the Retina in Age-Matched Wild Type and CCR2 Deficient Mice
}

\author{
J.R. Megill, E.B. Janovitz, J.H. Lin, and B.D. Car
}

Discovery Toxicology, Pharmaceutical Research Institute, Bristol-Myers Squibb Company, PO Box 5400, Princeton, NJ, 08543-5400, USA

Disruption of monocyte chemotactic protein-1/chemokine receptor-2 (MCP-1/CCR2) interaction has been proposed as a potential target for anti-inflammatory therapy [1], but also as a model for the development of age-related macular degeneration (AMD) [2,3]. A comparative study of the ultrastructure of the retinas from age-matched C57 CCR2 +/+ (WT) and CCR2 -/- (KO) mice was conducted to determine whether early onset retinal degeneration is a feature of the CCR2 KO phenotype. The study primarily focused on the photoreceptor layer, retinal pigment epithelium (RPE), Bruch's membrane (BM), and choriocapillaris. Retinas from mice at 9, 12, and 16 months of age were examined by transmission electron microscopy (TEM) and no alterations in the ultrastructural morphology or BM thickness in CCR2 KO mice were observed. This finding suggests that retinal degeneration may not be a liability of pharmacologic CCR2 inhibition and contrasts with a previous report that also included the misidentification of the normal inner nuclear layer as degeneration of the outer nuclear layer [2].

\section{References}

[1] C.M. Brodmerkel et al., J Immunol., 8 (2005) 5370.

[2] J. Ambati et al., Nature Medicine. 9 (2003) 1390

[3] J.V. Forrester, Nature Medicine. 9 (2003) 1350 

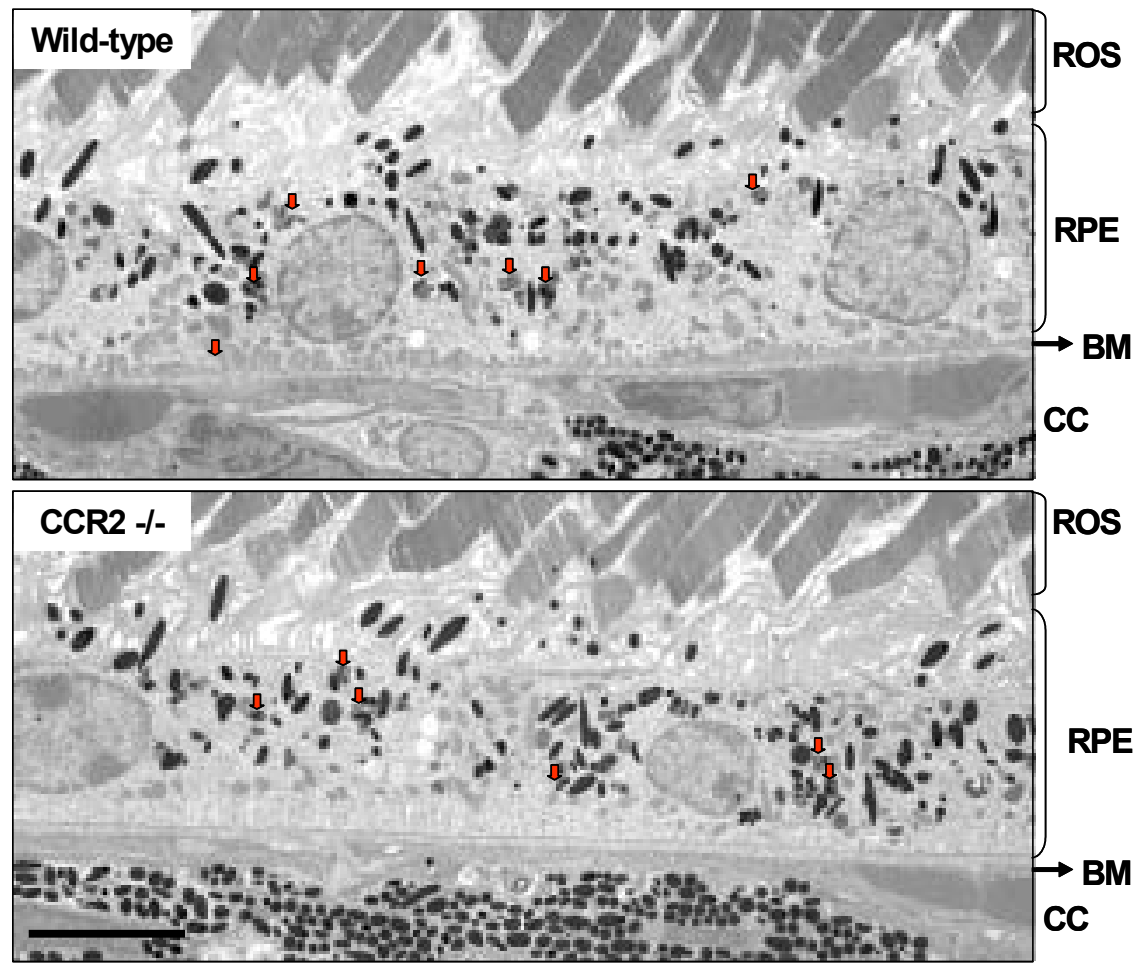

FIG. 1. TEM of the outer retina from 15-month old wild-type and CCR2-/- (KO) mice. There is no evidence of degeneration. and morphologic features are indistinguishable. Rod outer segments (ROS) were normally arrayed, Bruch's membrane (BM) thickness is uniform, and melanin granule size and distribution in the retinal pigment epithelium (RPE) and choriocapillaris (CC) are similar. Lipofucsin granules (arrows) present in RPE of both wild-type and CCR2 KO mice is a feature of the normal aging process. $\mathrm{Bar}=5 \mu \mathrm{M}$

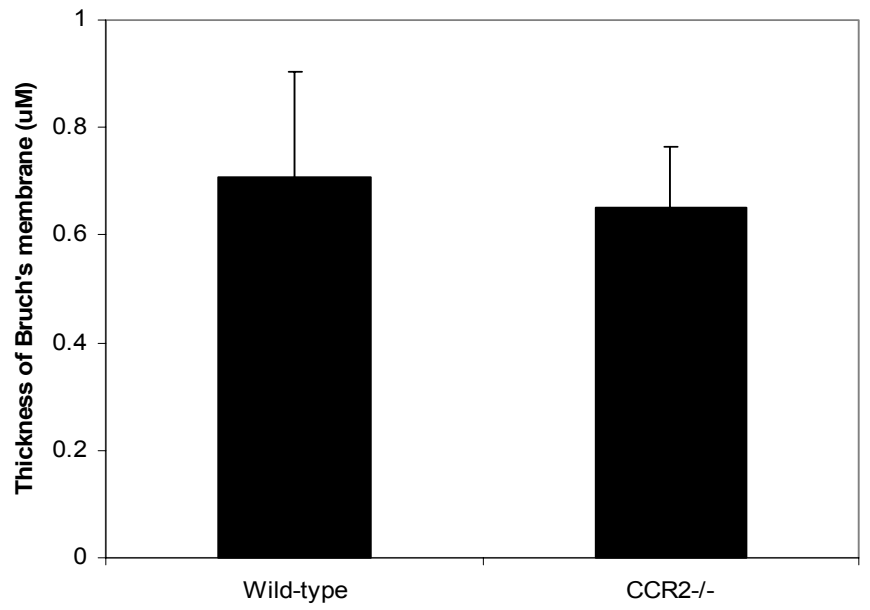

FIG. 2. Bruch's membrane thickness, determined by calibrating the measurement tool in Photoshop. There was no difference in Bruch's membrane thickness in 10-15 month old CCR2-/- (KO) mice $(\mathrm{N}=10)$ when compared to age-matched wild type mice $(\mathrm{N}=4)$. 